\title{
Gastrointestinal Soft Tissue Tumors
}

For details $\rightarrow$ see Chap. 74 .

(C) The Editor(s) (if applicable) and The Author(s), under exclusive license to 